\title{
Downlink Power Control: A Performance Booster for Full Duplex Cellular Networks
}

This paper was downloaded from TechRxiv (https://www.techrxiv.org).

\section{LICENSE}

CC BY 4.0

SUBMISSION DATE / POSTED DATE

$11-08-2021 / 13-08-2021$

\section{CITATION}

Mandali Kundu, Askar; Thazhathe Veetil, Sreejith (2021): Downlink Power Control: A Performance Booster for Full Duplex Cellular Networks. TechRxiv. Preprint. https://doi.org/10.36227/techrxiv.15147138.v1

$\mathrm{DOI}$ 


\title{
Downlink Power Control: A Performance Booster for Full Duplex Cellular Networks
}

\author{
Askar Mandali Kundu and Sreejith T V \\ Department of Electrical Engineering and Computer Science \\ Indian Institute of Technology, Bhilai, India 492015 \\ \{askarmk,sreejith\}@iitbhilai.ac.in
}

\begin{abstract}
Full duplex (FD) communication uses the same time-frequency resource elements for uplink and downlink transmissions. Compared to half-duplex (HD) systems, this improves the effective spectrum usage, but at the cost of additional self-interference and increased co-channel interference (CCI). Promising improvements in full duplex downlink performance are well established in the literature, but the improvement in uplink is marginal or even severely degraded due to strong down-link signals. This paper focuses on managing CCI in an FD network by spatially controlling downlink and uplink transmit powers. We show that uplink performance can be improved through the introduction of downlink power control, but this slightly reduces downlink performance. We introduce a distance-based user scheduling to address this reduction. Furthermore, we have also shown that optimal overall performance can be achieved by designing appropriate uplink and downlink power control factors.
\end{abstract}

\section{Index Terms}

Full duplex, uplink power control, downlink power control, interference management, user scheduling, stochastic geometry.

\section{INTRODUCTION}

The evolution of cellular networks from $4 \mathrm{G}$ to $5 \mathrm{G}$ has resulted in a steep increase in required data traffic [1], [2]. This will be further increased in 6G [3], [4]. Full duplex (FD) is considered one of the potential technologies to address this increased traffic demand. FD systems use the same time-frequency resource elements for downlink (DL) and uplink (UL) transmissions and 
thus double the spectrum usage compared to traditional half-duplex (HD) systems. However, simultaneous DL-UL transmissions on the same frequency increase interference and limit expected FD gains [5], [6].

The interferences are of three types, (i) self interference (SI): interference from the transmitter of a communication device to its own receiver, (ii) same cell interference (SCI): interference from other users of the same cell, and (iii) other cell interference (OCI): signals from BSs and users of other cells. Even though various signal processing techniques have emerged to cancel the SI [7]-[9], the bottleneck for FD performance in cellular systems is co-channel interference (SCI and OCI) from other uplink and downlink users. In this paper, we are interested in investigating the achievable rate gains by using spatially controlled transmit powers.

\section{A. Motivation and Related Works}

In the context of cellular systems, FD performances in the homogeneous networks are considered in [6], [10]. FD in heterogeneous networks is considered in [11]-[13]. These works show notable improvements in downlink performance while improvements in the uplink are marginal. However, these studies model BSs and UE locations as independent Poisson point processes, without taking into account the spatial dependencies of users and BSs.

In traditional HD cellular systems, UEs employ fractional power control in the uplink [14], and thus the transmit power of a user will be a function of its distance from the serving BS. This makes the transmit powers of UEs have spatial dependencies with the BSs, i.e., with this scheme, an UE near the BS will transmit with a lower power than a UE at the cell edge. Recently, models based on cluster processes are proposed for cellular system analysis to incorporate this dependency, [15]-[17].

The Thomson cluster process-based model is used in [18], to study the DL performance of FD networks, but uplink power control is not considered. In [19], the FD cellular network with uplink power control is analyzed using the Matèrn cluster process (MCP) and demonstrated severe reductions in mean and cell edge rate of uplink users due to high DL interference. The results also indicate that slight improvements in mean rate can be achieved by network densification with low-power BSs, nevertheless, cell edge rates are severely degraded. The MCP based model is also used in [20], which analyzed DL and UL performance with uplink power control. In order to minimize interference, not all BSs are permitted to use the full bandwidth of the operation 
and a minimum separation between BSs is imposed to use the same frequencies. This is similar to the concept of fractional frequency reuse and is contradictory to the fundamental principle of $\mathrm{FD}$, which is the maximum reuse of spectral resources.

In [21], the problem of user scheduling in FD cellular networks is considered to minimize inter-user interference. A single cell system with multiple users is considered in [22], [23] and proposes an algorithm for scheduling user pairs, but DL interference from other BSs is not taken into account. In addition, these works ignore UL power control. We believe that DL interference mitigation is as important as inter-user interference mitigation. In this paper, we consider the FD cellular network employing power control mechanisms in DL and UL. Our findings show that with a marginal trade-off in DL performance, we can improve UL performance and achieve most of the benefits of FD by controlling DL and UL transmit powers. We consider a distance-based user scheduling to cover this compromise in DL performance.

\section{B. Main focuses and Contributions}

In this paper, we analyze coverage and rate in FD cellular systems for three different power control scenarios. The main contributions are as follows:

- Fixed power downlink and uplink: In section III, we analyzed an FD cellular network with constant uplink and downlink power. We derived expressions for SINR distributions for downlink and uplink. Although this scheme is not practically relevant, it provides the basic analytical framework for later sections of the paper.

- FD in the traditional cellular system with fractional uplink power control: With derived analytical coverage probabilities and rates, we have shown that the uplink users in the traditional cellular networks cannot survive with FD. An upper bound for uplink coverage is also provided.

- Spatial power control in downlink and uplink: With the proposed downlink power control scheme and the conventional fractional uplink power control, we have shown that uplink users can be rescued in FD cellular networks. We also discussed the optimization and tradeoff between downlink and uplink performance. We have also shown that downlink and uplink coverages can be elevated with a distance-based user scheduling. 


\section{Organization of the paper}

In Section II, the system model is provided. In Section III, the traditional cellular network with FD is analyzed and the challenges of employing FD are discussed. In Section IV, downlink power control is introduced, and in Section V optimality of downlink and uplink power control is discussed and proposed a distance-based user scheduling. In Section VI the paper is concluded.

\section{System MODEL}

In this section, we provide a mathematical model of the cellular system that will be used in the subsequent analysis.

Network Model: The BS locations are distributed according to a homogeneous Poisson Point Process (PPP), $\Phi_{b}$, with density $\lambda_{b}$. The locations of the UEs are assumed to form a Matèrn Cluster Process (MCP) centred at the BS with a cell radius, R. Fig.1 shows a snapshot of the system model. Each BS schedules and allocates time-frequency resource elements to each of its UEs. In each cell, we assume a downlink UE and an uplink UE sharing the same resources (as has been allocated by the serving BS), which leads to cross-talk among the uplink and downlink paths. The limitation of this model is the overlapping of clusters of adjacent points of PPP in dense networks with large clusters. The probability of this overlapping of clusters can be reduced by choosing the density of the BSs, $\lambda_{b}=1 / \pi R^{2}$, i.e., the average number of BSs in a circle of radius $R$ is 1 .

Channel and path loss model: The channel between any two nodes is assumed to have Rayleigh fading with AWGN noise of variance $\sigma^{2}$. The pathloss is assumed to be exponential with the parameter, $\alpha$, i.e., the power received at a distance $x$ from a unit power transmitter will be $l(x)=x^{-\alpha}$. We assume $\alpha=4$ throughout the paper, unless otherwise specified.

Received signal and interference: We assume each BS serves two users, one in the downlink and the other in the uplink. The downlink user of BS, $x \in \phi_{b}$, is denoted by $d(x)$ and uplink user by $u(x)$. We use $h_{x_{1}, x_{2}}$ and $r_{x_{1}, x_{2}}$ respectively to denote channel and distance from node $x_{1}$ to node $x_{2}$. The signal transmitted by a node $x$ is represented as $a_{x}$. Then, the received signal at the downlink user, $d(\hat{o})$, associated with $\mathrm{BS}, \hat{o}$, is given by

$$
y_{d(\hat{o})}=\frac{h_{\hat{o}, d(\hat{o})} a_{\hat{o}}}{\sqrt{r_{\hat{o}, d(\hat{o})}^{-\alpha}}}+\frac{h_{u(\hat{o}), d(\hat{o})} a_{u(\hat{o})}}{\sqrt{r_{u(\hat{o}), d(\hat{o})}^{-\alpha}}}+\underbrace{\sum_{x \in \phi_{b} \backslash \hat{o}}\left(\frac{h_{x, d(\hat{o})} a_{x}}{\sqrt{r_{x, d(\hat{o})}^{-\alpha}}}+\frac{h_{u(x), d(\hat{o})} a_{u(x)}}{\sqrt{r_{u(x), d(\hat{o})}^{-\alpha}}}\right)}_{\text {Other cell interference }} .
$$




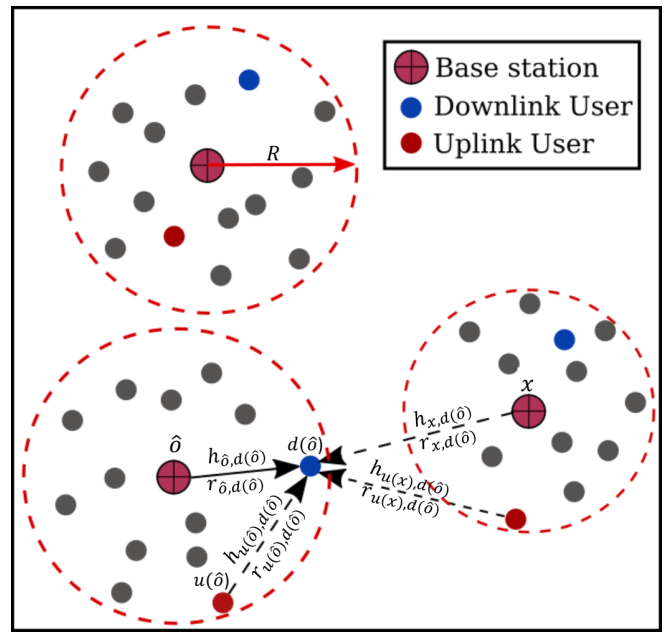

Fig. 1: A snapshot of the network. The UEs are distributed in circles of radius $R$ around each parent base station. A downlink UE (blue dot) of the cell will be served by the parent BS of its cell; uplink UE(s) allocated in the same resources are marked in red.

The first term in the RHS of (3) is the desired signal and the second term is the same cell uplink interference. The interference from other cells includes interferences from downlink BSs as well as from uplink users which are given in the third term.

Similarly, the uplink signal received at $\hat{o}$, can be written as,

$$
y_{\hat{o}}=\frac{h_{u(\hat{o}), \hat{o}} a_{u(\hat{o})}}{\sqrt{r_{u(\hat{o}), \hat{o}}^{-\alpha}}}+\underbrace{\sum_{x \in \phi_{b} \backslash \hat{o}}\left(\frac{h_{x, \hat{o}} a_{x}}{\sqrt{r_{x, \hat{o}}^{-\alpha}}}+\frac{h_{u(x), \hat{o}} a_{u(x)}}{\sqrt{r_{u(x), \hat{o}}^{-\alpha}}}\right)}_{\text {Other cell interference }} .
$$

In this paper, we assume complete cancellation of self-interference, which is an optimistic assumption and justifiable considering the recent efforts of researchers to minimize the selfinterference [7]-[9].

Distance to the serving BS: Since the UEs are distributed uniformly in each cell around the BS, the probability density function (PDF) of the distance to serving BS is given by [17],

$$
f_{R}(r)=\frac{2 r}{R^{2}}, \quad 0 \leq r \leq R
$$

Distance between two random users of same cells: The users of a cell are distributed uniformly in a circle of radius $R$. The PDF of the distance $l$ between two such points in a circle of radius 
$R$ is given by [24],

$$
f_{L}(l)=\frac{4 l}{\pi R^{2}}\left(\cos ^{-1}\left(\frac{l}{2 R}\right)-\frac{l}{2 R} \sqrt{1-\left(\frac{l}{2 R}\right)^{2}}\right), \quad 0 \leq l \leq 2 R .
$$

Downlink and Uplink Power Control: We consider three different downlink and uplink power scenarios in our analysis. In section III-A we assume a fixed downlink power, i.e., $\mathbb{E}\left[\left|a_{x}\right|^{2}\right]=P_{d}$ and fixed uplink power, all UEs are transmitting with constant power regardless of their distance from the tagged BS, i.e., $\mathbb{E}\left[\left|a_{x}\right|^{2}\right]=P_{u}$. In section III-B we assume a fixed downlink power but in the uplink, we consider the fractional power control. While in section IV we consider transmissions with fractional power control both in downlink and uplink.

Notations: The following functions frequently occur in our analysis and for convenience, we use the following notations.

$$
\begin{gathered}
\Lambda_{\lambda, \alpha}^{T} \triangleq \frac{2 \pi^{2} \lambda \csc \left(\frac{2 \pi}{\alpha}\right) T^{\frac{2}{\alpha}}}{\alpha} . \\
F_{s}^{1}(z) \triangleq{ }_{1} F_{1}\left(\frac{1}{s} ; 1+\frac{1}{s} ;-z\right) . \\
F_{s}^{2}(z) \triangleq{ }_{2} F_{1}\left(1, \frac{1}{s} ; 1+\frac{1}{s} ;-z\right) .
\end{gathered}
$$

\section{Challenges in Employing FD in Conventional Cellular Networks}

In this section, we provide the analysis of downlink and uplink coverage probabilities in conventional cellular networks. The average achievable rate and rate CDF (cumulative distribution function) can be derived from the coverage probability. We begin with constant power both in uplink and downlink. Even though the constant power assumption in uplink is not relevant for the cellular network, this will give a basic framework for our analysis in the following sections.

\section{A. Case I : Full Duplex with constant downlink and uplink power}

1) Downlink Coverage: For a downlink user, the interfering signals are from the uplink user of the same cell and uplink and downlink signals from the other cells as in equation (3). Conditioned on $r_{\hat{o}, d(\hat{o})}=r_{d}, r_{u(\hat{o}), d(\hat{o})}=l$, the SINR of the downlink user is given by

$$
\left.\operatorname{SINR}\right|_{r_{d}, l}=\frac{P_{d}\left|h_{\hat{o}}\right|^{2} r_{d}^{-\alpha}}{\sigma^{2}+P_{u}\left|h_{u}\right|^{2} l^{-\alpha}+I_{O B}(\hat{o})+I_{O U}(\hat{o})},
$$

where, $h_{\hat{o}}=h_{\hat{o}, d(\hat{o})}, h_{u}=h_{u(\hat{o}), d(\hat{o})}, I_{O B}(\hat{o})=\sum_{x \in \phi_{b} \backslash \hat{o}} P_{d}\left|h_{x, d(\hat{o})}\right|^{2} r_{x, d(\hat{o})}^{-\alpha}$ is the downlink interference power from other BSs and $I_{O U}(\hat{o})=\sum_{x \in \phi_{b} \backslash \hat{o}} P_{u}\left|h_{u(x), d(\hat{o})}\right|^{2} r_{u(x), d(\hat{o})}^{-\alpha}$ is the uplink interference power 
from users of other BSs. Then, the coverage probability (the cumulative distribution of the SINR) is given by the following theorem.

Theorem 1. Coverage probability for a downlink user in full duplex cellular network with constant uplink and downlink power is given by

$$
P_{c, D L}(T)=\mathbb{E}_{r_{d}, l}\left[\frac{e^{-\frac{T r_{d}^{\alpha} \sigma^{2}}{P_{d}}-\Lambda_{\alpha, \lambda}^{T} r_{d}^{2}\left(1+\left(\frac{P_{u}}{P_{d}}\right)^{\frac{2}{\alpha}}\right)}}{1+\frac{T P_{u} r_{d}^{\alpha}}{P_{d} l^{\alpha}}}\right],
$$

the expectation is over the random variables $r_{d}$ and $l$ for which the pdfs are given in (5) and (6).

Proof. The coverage probability, conditioned on $r_{d}$ and $l$ is given by,

$$
\begin{aligned}
& \left.P_{c, D L}(T)\right|_{r_{d}, l}=\mathbb{P}\left[\operatorname{SINR}>\left.T\right|_{r_{d}, l}\right], \\
& =\mathbb{P}\left[\frac{P_{d}\left|h_{\hat{o}}\right|^{2} r_{d}^{-\alpha}}{\sigma^{2}+P_{u}\left|h_{u}\right|^{2} l^{-\alpha}+I_{O B}(\hat{o})+I_{O U}(\hat{o})}>T\right], \\
& \stackrel{(a)}{=} \mathbb{E}\left[e^{-\frac{T r_{d}^{\alpha}}{P_{d}}\left[\sigma^{2}+P_{u}\left|h_{u}\right|^{2} l^{-\alpha}+I_{O B}(\hat{o})+I_{O U}(\hat{o})\right]}\right], \\
& \stackrel{(b)}{=}\left(\frac{e^{-\frac{T r_{d}^{\alpha} \sigma^{2}}{P_{d}}}}{1+\frac{P_{u} T r_{d}^{\alpha}}{P_{d} l^{\alpha}}}\right) \mathcal{L}_{I_{O B}}\left(\frac{T r_{d}^{\alpha}}{P_{d}}\right) \mathcal{L}_{I_{O U}}\left(\frac{T r_{d}^{\alpha}}{P_{d}}\right),
\end{aligned}
$$

where (a) follows by noting that $\left|h_{\hat{o}}\right|^{2}$ is exponential random variable and using its tail probability, (b) is by using the definition of Laplace transform for interference, i.e., $\mathcal{L}_{I}(s)=\mathbb{E}\left(e^{-s I}\right)$. In (b) we also assume that the interference from other BSs and their users are independent, which is not exact. The derivation of $\mathcal{L}_{I}(s)$ follows the similar procedure of [17], [25] and is given below.

$$
\begin{aligned}
\mathcal{L}_{I_{O B}}(s) & =\mathbb{E}_{\Phi_{b}}\left[\exp \left(-s \sum_{x \in \phi_{b} \backslash \hat{o}} P_{d}\left|h_{x, d(\hat{o})}\right|^{2} r_{x, d(\hat{o})}-\alpha\right)\right], \\
& =\exp \left(-2 \pi \lambda P_{d} \int_{0}^{\infty} \frac{s r_{x, d(\hat{o})}^{-\alpha}}{1+s r_{x}^{-\alpha}} r_{x} \mathrm{~d} r_{x, d(\hat{o})}\right), \\
& =\exp \left(-\frac{2 \pi^{2} \lambda P_{d} \csc \left(\frac{2 \pi}{\alpha}\right) s^{\frac{2}{\alpha}}}{\alpha}\right) .
\end{aligned}
$$

Consider a realization $\phi_{b}$, the tagged users are uniformly and randomly chosen from a disc of radius $R$. We can see that the locations of uplink users will be similar to a PPP of density $\lambda$, 
even though their locations are not independent of $\phi_{b}$, we assume these other cell users as a PPP of density $\lambda_{b}$. Using extensive simulations, we confirmed that this assumption does not deviate analytical results from the actual simulation of MCP. A similar assumption can be seen in [26]. Using this assumption, the Laplace transform of other cell user interference is given by

$$
\begin{aligned}
\mathcal{L}_{O U}(s) & =\mathbb{E}_{\Phi_{b}}\left[\exp \left(-s P_{u} \sum_{x \in \Phi_{b} \backslash \hat{o}}\left|h_{u(x), d(\hat{o})}\right|^{2} r_{u(x), d(\hat{o})}{ }^{-\alpha}\right)\right], \\
& =\exp \left(-\frac{2 \pi^{2} \lambda \csc \left(\frac{2 \pi}{\alpha}\right) P_{u}^{\frac{2}{\alpha}} s^{\frac{2}{\alpha}}}{\alpha}\right) .
\end{aligned}
$$

The proof is complete by substituting (13) and (14) in (12) and taking the expectation over $r_{d}$ and $l$.

An observation and validation: For the noiseless scenario, i.e., when $\sigma=0$, we can formulate a simple and closed form expression for downlink coverage probability in half duplex system by substituting $P_{u}=0$ in (11) and taking expectation over $r_{d}$,

$$
P_{c, D L}^{H D}(T)=\frac{\alpha\left(1-e^{-\frac{2 \pi^{2} \lambda R^{2} \csc \left(\frac{2 \pi}{\alpha}\right) T^{\frac{2}{\alpha}}}{\alpha}}\right)}{2 \pi^{2} \lambda R^{2} \csc \left(\frac{2 \pi}{\alpha}\right) T^{\frac{2}{\alpha}}} .
$$

Now by substituting our assumption $\lambda=\frac{1}{\pi R^{2}}$, we can see that the coverage probability of HD downlink is independent of $\lambda$ and $P_{d}$. The same observation can be seen for PPP based half duplex networks with nearest neighbor connectivity, [25].

In Fig.2, we can see that the coverage in MCP with $\lambda=1 / \pi R^{2}$ and PPP of the same density, are close but MCP being the lower bound. This is because of two reasons, (i) in MCP based networks, we can limit the overlapping of clusters by assuming $\lambda=1 / \pi R^{2}$, the average number of BSs in a circle of radius $R$ is 1 , but it does not ensure nearest connectivity and hence there is a positive probability for an interfering BS nearer than the tagged BS, (ii) reducing the probability of overlapping cluster can create voids in the coverage region. Hence, the results in this paper are lower bounds for PPP-based nearest BS connectivity networks. In PPP based nearest connectivity model, the exact characterization of inter-user distance inside a cell (random voronoi cell) is difficult and the analysis of network level parameter will be cumbersome. Hence, we use the proposed simple model and we have compared our results with PPP based network through simulations and found the behavior of network performances are similar, MCP being the lower bound. 


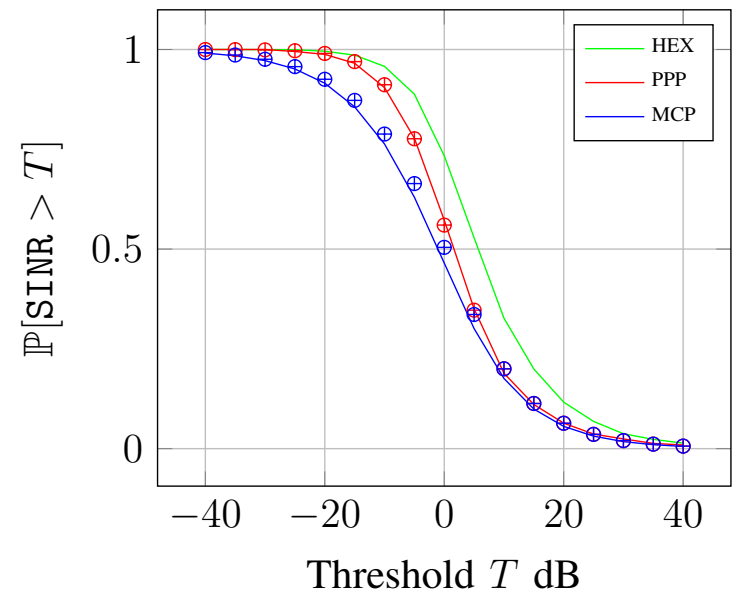

Fig. 2: Downlink Coverage probability of HD network for Hexogonal, PPP and proposed MCP model with $P_{d}=40 \mathrm{dBm}$ and cell radius, $R=200 \mathrm{~m}$. Simulations are marked by solid lines and theoretical results by respective colored $\oplus$ mark.

2) Uplink Coverage: Conditioned on the tagged user distance $r_{u}$ from the typical BS, $\hat{o}$, the SINR at the BS in the uplink is given by,

$$
\left.\operatorname{SINR}\right|_{r_{u}}=\frac{P_{u}\left|h_{u(\hat{o}), \hat{o}}\right|^{2} r_{u}^{-\alpha}}{\sigma^{2}+\sum_{x \in \Phi_{b}^{\prime}}\left(P_{d}\left|h_{x, \hat{o}}\right|^{2} r_{x, \hat{o}}^{-\alpha}+P_{u}\left|h_{u(x), \hat{o}}\right|^{2} r_{u(x), \hat{o}}^{-\alpha}\right)},
$$

where $P_{d}$ and $P_{u}$ are the constant uplink and downlink powers. The coverage probability is given by the following theorem.

Theorem 2. Coverage probability for a uplink user in full duplex cellular network with constant uplink and downlink power is given by

$$
\mathbb{P}_{c}(T)=\mathbb{E}_{r_{u}}\left[e^{-\frac{\sigma^{2} T_{r}^{\alpha}}{P_{u}}-\Lambda_{\lambda, \alpha}^{T} r_{u}^{2}\left(1+\left(\frac{P_{d}}{P_{u}}\right)^{\frac{2}{\alpha}}\right)}\right],
$$

where the expectation is over the random variables $r_{u}$ for which pdf is given in (5)

Proof. Follows the similar procedure of Theorem. 1

As cellular networks are transitioning from noise-limited low-dense regime to high-dense interference-limited regime, interference limited scenario is worth analyzing. The next corollary provides a simple closed form expression for coverage of an uplink user in interference limited scenario. 
Corollary 1. For interference limited scenario $\left(\sigma^{2}=0\right)$, the coverage probability of a typical uplink user is given by,

$$
\mathbb{P}_{c}(T)=\frac{\left.1-e^{-\Lambda_{\lambda, \alpha}^{T} R^{2}\left(1+\left(\frac{P_{d}}{P_{u}}\right)^{\frac{2}{\alpha}}\right.}\right)}{\Lambda_{\lambda, \alpha}^{T} R^{2}\left(1+\left(\frac{P_{d}}{P_{u}}\right)^{\frac{2}{\alpha}}\right)} .
$$

Proof. Substitute $\sigma^{2}=0$ in (17), then by taking the expectation over $r_{u}$.

We can see that the coverage probability can be increased either by reducing $P_{d}$ and/or by increasing $P_{u}$, but increasing $P_{u}$ beyond some limit is not possible in the cellular networks since, the users are power-limited devices. As seen in the downlink, the coverage in uplink is also independent of $\lambda$.

In the next section, we will discuss the FD coverage in a conventional cellular network with uplink power control, where we can see that the coverage probabilities are depending on $\lambda$.

\section{B. Case II : Full Duplex with constant power in downlink and fractional power control in uplink}

We consider users employing the distance-dependent fractional uplink power control as in [14], [27], [28]. In this scheme the transmit power of a user in uplink is set according to,

$$
P_{u}^{\epsilon}(P L)=\min \left(P_{\max }^{u}, P_{u}^{\circ}+\epsilon P L\right) \quad \mathrm{dBm},
$$

where $\epsilon \in[0,1]$ and $P_{u}^{\circ}$ are the power control factors, designed to achieve a target SINR, $P_{\max }^{u}$ is the ceiling for uplink power, and $P L$ is the estimated pathloss. The nominal values of $P_{u}^{\circ}$, $-127<P_{u}^{\circ} \leq-64 \mathrm{dBm}$, [27]. This transmission scheme compensates for the pathloss and tries to maintain the SINR needed to establish a connection with the serving BS. So a UE near to the BS will be transmitting at lower power and a user far from BS will be transmitting at higher power. This makes the spatial distribution of interference to be very different from that of transmission without power control. A preliminary study on this case is presented in [19].

1) Downlink Coverage: Conditioned on $r_{\hat{o}, d(\hat{o})}=r_{d}, r_{\hat{o}, u(\hat{o})}=r_{u}$ and inter user distance, $r_{d(\hat{o}), u(\hat{o})}=l$, from (4), the SINR can be given as

$$
\left.\operatorname{SINR}\right|_{r_{d}, r_{u}, l}=\frac{P_{d}\left|h_{\hat{o}}\right|^{2} r_{d}{ }^{-\alpha}}{\sigma^{2}+P_{u}^{\epsilon}\left(r_{u}\right)\left|h_{u}\right|^{2} l^{-\alpha}+I_{O B}+I_{O U}(\epsilon)},
$$

where $P_{u}^{\epsilon}\left(r_{u}\right)$ is the uplink power of a user when it is at a distance $r_{u}$ from its tagged BS, $I_{O B}=\sum_{x \in \phi_{b} \backslash \hat{o}} P_{d}\left|h_{x, d(\hat{o})}\right|^{2} r_{x, d(\hat{o})}^{-\alpha}$ and $I_{O U}(\epsilon)=\sum_{x \in \phi_{b} \backslash \hat{o}} P_{u}^{\epsilon}\left(r_{u(x), x}\right)\left|h_{u(x), d(\hat{o})}\right|^{2} r_{u(x), d(\hat{o})}^{-\alpha}$. The downlink coverage probability is given in the next theorem. 
Theorem 3. The downlink coverage probability in an FD cellular network when the uplink users are employed with fractional power control is given by,

$$
\mathbb{P}_{c, D L}^{\epsilon}(T) \approx \mathbb{E}_{r_{d}, l}\left[F_{\epsilon}^{1}\left(\Lambda_{\lambda, \alpha}^{T}\left(\frac{P_{u}^{\circ}}{P_{d}}\right)^{\frac{2}{\alpha}} R^{2 \epsilon} r_{d}^{2}\right) F_{\frac{\alpha \epsilon}{2}}^{2}\left(\frac{P_{u}^{\circ} T R^{\alpha \epsilon} r_{d}^{\alpha}}{P_{d} l^{\alpha}}\right) e^{-\frac{\sigma_{n}^{2} T r_{d}^{\alpha}}{P_{d}}-\Lambda_{\lambda, \alpha}^{T} r_{d}^{2}}\right],
$$

where the expectation is over the random variables $r_{d}$ and $l$, and their pdf is given in (5) and (6).

Proof. Conditioned on $r_{d}, r_{u}, r_{u(x), x}$ and $l$, the coverage probability is given by,

$$
\begin{aligned}
& \left.P_{c}^{\epsilon}(T)\right|_{r_{d}, r_{u}, l, r_{u(x), x}}=\mathbb{P}\left[\left.\operatorname{SINR}\right|_{r_{d}, r_{u}, l, r_{u(x), x}}>T\right], \\
& =\mathbb{P}\left[\frac{P_{d}\left|h_{\hat{o}, d(\hat{o})}\right|^{2} r_{d}^{-\alpha}}{\sigma^{2}+P_{u}^{\epsilon}\left(r_{u}\right)\left|h_{u(\hat{o}), d(\hat{o})}\right|^{2} l^{-\alpha}+I_{O B}+I_{O U}(\epsilon)}>T\right], \\
& \stackrel{(a)}{=} \exp \left(-\frac{T r_{d}^{\alpha} \sigma_{n}^{2}}{P_{d}}\right) \underbrace{\left(\frac{1}{1+\frac{P_{u}^{\epsilon}\left(r_{u}\right) T r_{d}^{\alpha}}{P_{d} l^{\alpha}}}\right)}_{T_{0}} \mathcal{L}_{I_{O B}}\left(T r_{d}^{\alpha}\right) \\
& \times \underbrace{\exp \left(-\frac{2 \pi^{2} \lambda \csc \left(\frac{2 \pi}{\alpha}\right) T^{\frac{2}{\alpha}} r_{u(x), x}^{2 \epsilon} r_{d}^{2}}{\alpha}\left(\frac{P_{u}^{\circ}}{P_{d}}\right)^{\frac{2}{\alpha}}\right)}_{T_{1}},
\end{aligned}
$$

where (a) follows by noting that $\left|h_{\hat{o}, d(\hat{o})}\right|^{2}$ is exponential distributed random variable and using its tail probability, then using the Laplace transform of interference.

The power control given in equation (19) can be restated in linear scale as, $P_{u}^{\epsilon}\left(r_{u}\right)=$ $\min \left(P_{\max }^{u}, P_{u}^{\circ} r_{u}^{\alpha \epsilon}\right)$. Assume the user adjust its uplink power based on the long term average RSRP, then

$$
P_{u}^{\epsilon}\left(r_{u}\right)= \begin{cases}P_{u}^{\circ} r_{u}^{\alpha \epsilon} & 0 \leq r_{u} \leq \eta \\ P_{\max }^{u} & \eta<r_{u} \leq R\end{cases}
$$

where $\eta=\left(P_{\max }^{u} / P_{u}^{\circ}\right)^{\frac{1}{\alpha \epsilon}}$. The user power is upper limited by $P_{\max }^{u}$ when distance $r_{u}>\eta$.

The expectation in (21) has to be taken along $r_{d}, r_{u}, r_{u(x), x}, l$, in which the term $T_{0}$ depends on $r_{u}$ and $T_{1}$ depends on $r_{u(x), x}$.

By substituting (22) in $T_{0}$, we have

$$
\begin{aligned}
\mathbb{E}_{r_{u}}\left(T_{0}\right) & =\int_{r_{u}=0}^{\eta} \frac{f_{R_{u}}\left(r_{u}\right) \mathrm{d} r_{u}}{1+P_{u}^{\circ} r_{u}^{\alpha \epsilon} \operatorname{Tr}_{d}^{\alpha} / P_{d} l^{\alpha}}+\int_{r_{u}=\eta}^{R} \frac{f_{R_{u}}\left(r_{u}\right) \mathrm{d} r_{u}}{1+P_{m a x}^{u} T r_{d}^{\alpha} / P_{d} l^{\alpha}}, \\
& \stackrel{(a)}{\approx} \int_{r_{u}=0}^{R} \frac{2 r_{u} \mathrm{~d} r_{u}}{R^{2}\left(1+P_{u}^{\circ} r_{u}^{\alpha \epsilon} T r_{d}^{\alpha} / P_{d} l^{\alpha}\right)},
\end{aligned}
$$




$$
={ }_{2} F_{1}\left(1, \frac{2}{\alpha \epsilon} ; \frac{\alpha \epsilon+2}{\alpha \epsilon} ;-\frac{P_{u}^{\circ} \operatorname{Tr}_{d}^{\alpha} R^{\alpha \epsilon}}{P_{d} l^{\alpha}}\right)
$$

where (a) is by noting that for moderate and high dense networks, the power saturating distance, $\eta>R$, and hence second term is neglected.

The uplink user $u(x)$ is a random point (uniformly distributed) in $\mathcal{B}(x, R)$, pdf of the distance between $u(x)$ and its tagged $\mathrm{BS}, x \in \phi_{b}$, is given by (5). Now expectation of $T_{1}$ is given by,

$$
\begin{aligned}
\mathbb{E}_{r_{u(x), x}}\left(T_{1}\right) & =\int_{u=0}^{R} \exp \left(-\frac{2 \pi^{2} \lambda \csc \left(\frac{2 \pi}{\alpha}\right) T^{\frac{2}{\alpha}} u^{2 \epsilon} r_{d}^{2}}{\alpha}\left(\frac{P_{u}^{\circ}}{P_{d}}\right)^{\frac{2}{\alpha}}\right) \frac{2 u}{R^{2}} \mathrm{~d} u, \\
& ={ }_{1} F_{1}\left(\frac{1}{\epsilon} ; \frac{\epsilon+1}{\epsilon} ;-\Lambda_{\lambda, \alpha}^{T}\left(\frac{P_{u}^{\circ}}{P_{d}}\right)^{\frac{2}{\alpha}} R^{2 \epsilon} r_{d}^{2}\right) .
\end{aligned}
$$

Substituting (23) and (24) in (21) will complete the proof.

2) Uplink Coverage: Conditioned that typical user is at a distance $r_{u}$ from the parent $\mathrm{BS}$, the SINR is given by

$$
\left.\operatorname{SINR}\right|_{r_{u}}=\frac{P_{u}^{\epsilon}\left(r_{u}\right)\left|h_{u(\hat{o}), \hat{o}}\right|^{2} r_{u}^{-\alpha}}{\sigma^{2}+I_{O B}^{U L}+I_{O U}^{U L}(\epsilon)},
$$

where $I_{O B}^{U L}=\sum_{x \in \Phi_{b} \backslash \hat{o}} P_{d}\left|h_{x, \hat{o}}\right|^{2} r_{x, \hat{o}}^{-\alpha}$ and $I_{O U}^{U L}(\epsilon)=\sum_{x \in \Phi_{b} \backslash \hat{o}} P_{u}^{\epsilon}\left(r_{u(\mathrm{x}), x}\right)\left|h_{u(x), \hat{o}}\right|^{2} r_{u(x), \hat{o}}^{-\alpha}$.

The coverage probability in uplink is given by the following theorem.

Theorem 4. Coverage probability for an uplink user in full duplex cellular network with fractional uplink power control and constant downlink power is given by

$$
\mathbb{P}_{c, U L}^{\epsilon}(T) \approx \mathbb{E}_{r_{u}}\left[F_{\epsilon}^{1}\left(\Lambda_{\lambda, \alpha}^{T} R^{2 \epsilon} r_{u}^{2(1-\epsilon)}\right) e^{\left.-\frac{\sigma^{2} T r_{u}^{\alpha(1-\epsilon)}}{P_{u}^{\circ}}-\Lambda_{\lambda, \alpha}^{T}\left(\frac{P_{d}}{P_{u}^{o}}\right)^{\frac{2}{\alpha}} r_{u}^{2(1-\epsilon)}\right],}\right.
$$

where the expectation is over the random variable $r_{u}$ and it's pdf is given in (5).

Proof. Follows the similar procedure of theorem (3).

The next corollary will provide a simple closed form upper bound for the uplink coverage probability in conventional networks. The upper bound is derived by neglecting the uplink user signals of others cells. This will be a tight bound since (i) the uplink signal powers (transmitted by users) are small compared to the downlink signal powers of BSs and (ii) other cell users are relatively at larger distances than the same cell users. 
Corollary 2. For interference limited scenario the coverage probability in unplink of a FD cellular network with fractional power control is upper bounded by,

$$
\mathbb{P}_{c, U L}^{\epsilon}(T)<{ }_{1} F_{1}\left(\frac{1}{1-\epsilon} ; \frac{2-\epsilon}{1-\epsilon} ;-\Lambda_{\lambda, \alpha}^{T}\left(\frac{P_{d}}{P_{u}^{\circ}}\right)^{\frac{2}{\alpha}} R^{2(1-\epsilon)}\right) .
$$

Proof. When $\sigma_{n}^{2}=0$ in (26) and taking expectation over $r_{u}$, we have

$$
\begin{aligned}
\mathbb{P}_{c, U L}^{\epsilon}(T) & \approx \frac{1}{1-\epsilon} \frac{\Gamma\left(\frac{1}{1-\epsilon}\right)-\Gamma\left(\frac{1}{1-\epsilon}, \Lambda_{\lambda, \alpha}^{T}\left(\frac{P_{d}}{P_{u}^{\circ}}\right)^{\frac{2}{\alpha}} R^{2(1-\epsilon)}\right)}{\left(\Lambda_{\lambda, \alpha}^{T}\left(\frac{P_{d}}{P_{u}^{\circ}}\right)^{\frac{2}{\alpha}} R^{2(1-\epsilon)}\right)^{\frac{1}{1-\epsilon}}}, \\
& \stackrel{(a)}{\approx} \frac{1}{1-\epsilon} \frac{\gamma\left(\frac{1}{1-\epsilon}, \Lambda_{\lambda, \alpha}^{T}\left(\frac{P_{d}}{P_{u}^{\circ}}\right)^{\frac{2}{\alpha}} R^{2(1-\epsilon)}\right)}{\left(\Lambda_{\lambda, \alpha}^{T}\left(\frac{P_{d}}{P_{u}^{\circ}}\right)^{\frac{2}{\alpha}} R^{2(1-\epsilon)}\right)^{\frac{1}{1-\epsilon}}}, \\
& \stackrel{(b)}{\approx}{ }_{1} F_{1}\left(\frac{1}{1-\epsilon} ; \frac{2-\epsilon}{1-\epsilon} ;-\Lambda_{\lambda, \alpha}^{T}\left(\frac{P_{d}}{P_{u}^{\circ}}\right)^{\frac{2}{\alpha}} R^{2(1-\epsilon)}\right),
\end{aligned}
$$

where (a) is using the property of incomplete gamma fuction, $\Gamma(x)-\Gamma(x, s)=\gamma(x, s)$ and (b) is by using the property of lower incomplete gamma function, $\gamma(x, s)=x^{-1} s^{x}{ }_{1} F_{1}(x, 1+x,-s)$.

\section{Numerical Results and Simulations}

We have used extensive Monte-Carlo simulations to verify the analytical results. We have simulated MCP and PPP based nearest connectivity models in a rectangular area of $100 \times 100$ sq. km. The downlink and uplink coverage probabilities are plotted for various configurations of $P_{d}, P_{u}$ and $\epsilon$ in Fig. 3 and we can see that the analytical results are closely matching with the simulations. We choose $P_{u}^{o}=-64 \mathrm{dBm}$ which is the maximum allowed in uplink transmission as per the standards [14]. We can see that by increasing $\epsilon$, uplink coverage probabilities are increasing with slights reductions in downlink coverage. However, it can also be seen that the coverage probability of uplink is almost $40 \mathrm{~dB}$ behind that of downlink, even with full power inversion $(\epsilon=1)$. For pathloss exponent $\alpha=3$ (low pathloss environment), the uplink coverage is further reduced to $60 \mathrm{~dB}$ below the downlink coverage.

Dependency of coverage probability with network density (inversely related to $R^{2}$ ) is illustrated in Fig. 4 for a fixed threshold, $T=-6 \mathrm{~dB}$. 


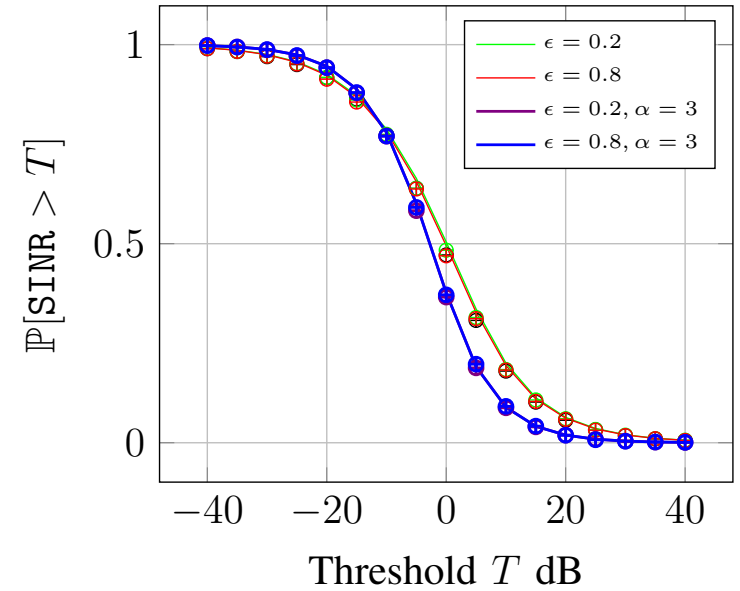

(a) Downlink Coverage

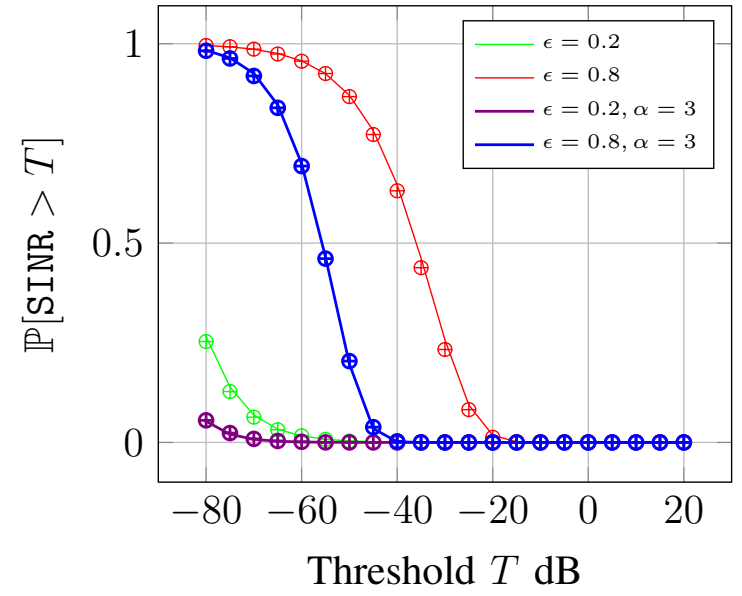

(b) Uplink Coverage

Fig. 3: Coverage probability of a typical user in FD network with uplink power control with fixed $P_{d}=40 \mathrm{dBm}, P_{u}^{\circ}=-64 \mathrm{dBm}$ and cell radius, $R=200 \mathrm{~m}$. Simulations are marked by respective colored $\oplus$.

\section{An important observation:}

- The downlink coverage probability is not independent of BS density in FD networks. This trend is different from that of conventional networks. This is because, with the fractional power control, the spatial characteristics of the user interference is changed, i.e., mathematically $\Lambda_{\lambda, \alpha}^{T}\left(\frac{P_{d}}{P_{u}^{o}}\right)^{\frac{2}{\alpha}} R^{2(1-\epsilon)}$ is a function of $R$ and $\epsilon$ and cannot be separated to $\lambda R^{2}$ form as in HD networks.

We can also see from Fig. 4 that the uplink coverage is almost impossible (0\%) in high dense networks ( $R<400 \mathrm{~m}$ ) and is slightly improved (to less than 18\%) by increasing $\epsilon$ in marginally dense networks $(R=1000 \mathrm{~m})$. The frequency reuse reduces the number of interfering BSs by fixed and planned frequency reuse patterns, which is similar (not exactly equivalent since frequency planning is not considered here) to BS density reduction in MCP based model. The results are not in favor of frequency reuse as the improvement in the uplink coverage is marginal with the reduction in the downlink coverage. This coverage improvement in uplink will also be balanced against the reduced bandwidth when considering the rate performance.

The main attraction of FD is the doubling of the spectrum usage. The effect of increased bandwidth utilization can be better understood by analyzing the rate profile. 


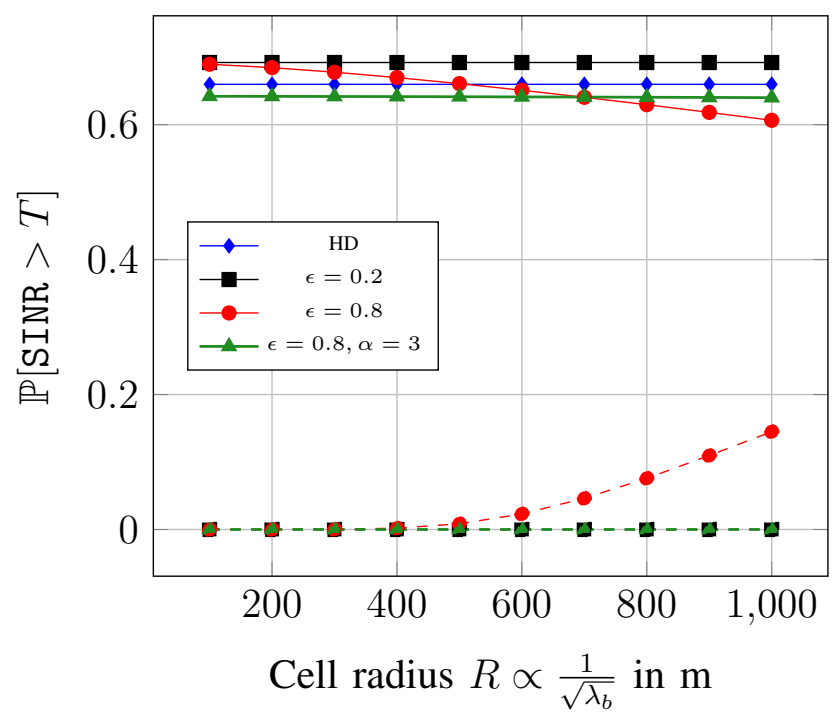

Fig. 4: Coverage probability of a typical user in an FD cellular network against different cell radius for various $\epsilon$ values at threshold SINR, $T=-6 \mathrm{~dB} . P_{u}^{\circ}=-64 \mathrm{dBm}, P_{d}=40 \mathrm{dBm}$. The solid curves for downlink and dashed curves for uplink.

\section{Rate Analysis}

We analyze the mean achievable rate and cell edge rate. The average rate $\mathbb{E}\left(R=\log _{2}(1+\right.$ SINR)) can be deduced from the SINR distribution using the property of positive random variables, $\mathbb{E}(X)=\int_{0}^{\infty} \mathbb{P}(X>t) \mathrm{d} t$. As per ITU, the cell edge rate is defined as the 5 percentile point of the cumulative distribution function $(\mathrm{CDF})$ of rate. The rate $\mathrm{CDF}, F_{C}(c)=\mathbb{P}(C \leq c)$, can also be obtained from the coverage probability.

The rate CDF for HD systems is given by,

$$
F_{C}^{H}(c)=\mathbb{P}\left[\log _{2}\left(1+\operatorname{SINR}_{H D}\right)<c\right]=1-P_{c}^{H D}(T)\left[2^{c}-1\right],
$$

where $c$ is the rate in bps/Hz. For FD systems, each unit of bandwidth is utilized twice and hence,

$$
F_{C}^{F}(c)=\mathbb{P}\left[2 \log _{2}\left(1+\operatorname{SINR}_{F D}\right)<c\right]=1-P_{c}^{F D}(T)\left[2^{\frac{c}{2}}-1\right]
$$

The rate profile is given in Table I for various combinations of $\epsilon$ and $P_{d}(\mathrm{dBm})$. From the table, we can see that average and cell edge rate is almost doubled in downlink of FD system, i.e., mean rate from 2.02 to $4.04 \mathrm{bps} / \mathrm{Hz}$ and cell edge rate from 0.0062 to $0.0125 \mathrm{bps} / \mathrm{Hz}$. This is true for low power BSs also $(23 \mathrm{dBm})$. The rate profile also reveals that the uplink users are 
severely affected by the strong interfering signals and the mean and cell edge rates are almost zero. The rate CDF's are plotted in Fig. 5, we can see that the rates are almost surely zero in the uplink, i.e., more than $90 \%$ of the users in the uplink are getting rates below $1 \mathrm{bps} / \mathrm{Hz}$, while $90 \%$ downlink users are above $15 \mathrm{bps} / \mathrm{Hz}$.

\begin{tabular}{|c|c|c|c|c|}
\hline \multirow{2}{*}{$\left(\epsilon, P_{d}\right)$} & \multicolumn{2}{|c|}{ Uplink } & \multicolumn{2}{c|}{ Downlink } \\
\cline { 2 - 5 } & $5 \%$ Rate & Mean Rate & $5 \%$ Rate & Mean Rate \\
\hline$(0.2,40)$ & $2.8 \times 10^{-11}$ & $2.8 \times 10^{-5}$ & 0.0125 & 4.04 \\
\hline$(0.8,40)$ & $4.1 \times 10^{-06}$ & $3.6 \times 10^{-3}$ & 0.0120 & 4.00 \\
\hline$(0.2,23)$ & $1.4 \times 10^{-09}$ & $3.3 \times 10^{-4}$ & 0.0125 & 4.04 \\
\hline$(0.8,23)$ & $2.0 \times 10^{-04}$ & $1.5 \times 10^{-1}$ & 0.0099 & 3.82 \\
\hline $\operatorname{HD}(0.2)$ & 0.005 & 2.27 & 0.0062 & 2.02 \\
\hline
\end{tabular}

TABLE I: Rate (bps/Hz) profile for various configurations of $\left(\epsilon, P_{d}\right)$ for cell radius $R=200 \mathrm{~m}$ and $P_{u}^{\circ}=-64 \mathrm{dBm}$.

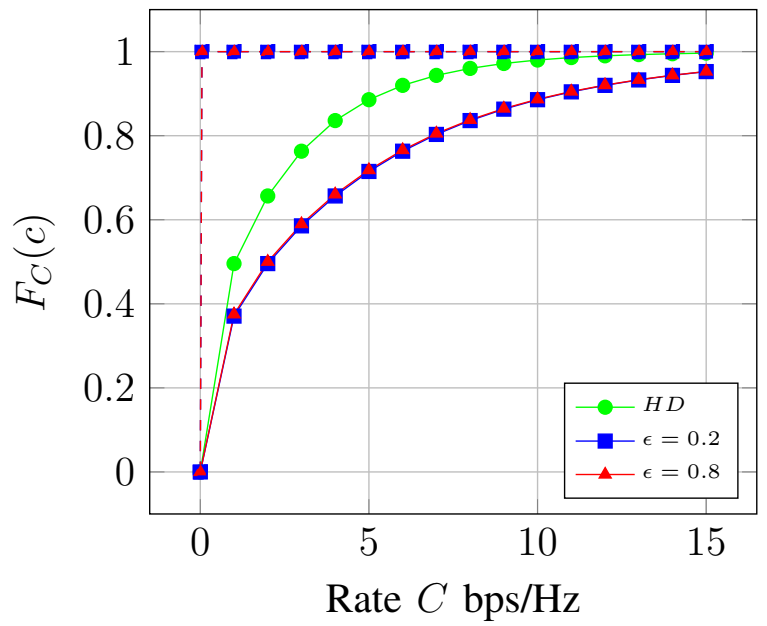

Fig. 5: Rate CDF of a typical user in FD with uplink power control for various $\epsilon$ with $P_{d}=40$ $\mathrm{dBm}, P_{u}^{\circ}=-64 \mathrm{dBm}$ and $R=200 \mathrm{~m}$. Solid curves for downlink and dashed curve of the respective color for uplink.

From the above discussions, we can see that the traditional cellular networks (with fixed downlink power and fractional uplink power) cannot survive with FD transmission because of the poor uplink performance in presence of strong co-channel downlink interference. We show in the next section that the uplink users can be rescued in cellular FD networks by appropriately designing 
the spatial characteristics of downlink powers, without sacrificing downlink performance to a large extend.

\section{Full DupleX With DOWNLINK AND UPLINK POWER CONTROL}

In this section, we introduce a fractional power control strategy in the downlink. We believe that there can be optimal power control mechanisms that perform better than our approach, nevertheless, our attempt is to provide a basic mathematical framework to analyze cellular networks with spatial power control in downlink and to show that the downlink power control can improve FD communication capabilities in cellular networks.

We consider a distance depended fractional power control scheme in downlink as given below,

$$
P_{d}^{\beta}(P L)=P_{d}^{\circ}+\beta P L \quad \mathrm{dBm},
$$

where $\beta \in[0,1]$ is the downlink power control factor, $P_{d}^{\circ}$ is the power parameter to ensure a minimum SINR and $P L$ is the estimated pathloss. The users employ traditional distance dependent fractional uplink power control as in (19).

1) Downlink Coverage: Conditioned on the typical downlink user distance from BS, $r_{d}$, uplink user distance, $r_{u}$, distacce between uplink and downlink users, $l$, the SINR is given by

$$
\begin{aligned}
& \left.\operatorname{SINR}\right|_{r_{d}, r_{u}, l} \\
& =\frac{P_{d}^{\beta}\left(r_{d}\right)\left|h_{\hat{o}}\right|^{2} r_{d}^{-\alpha}}{\sigma^{2}+P_{u}^{\epsilon}\left(r_{u}\right)\left|h_{u}\right|^{2} l^{-\alpha}+I_{O B}(\beta)+I_{O U}(\epsilon)},
\end{aligned}
$$

where $h_{\hat{o}}=h_{\hat{o}, d(\hat{o})}$ are $h_{u}=h_{u(\hat{o}), d(\hat{o})}$ are channels observed at downlink user of $\hat{o}, d(\hat{o})$, $I_{O B}(\beta)=\sum_{x \in \Phi_{b} \backslash \hat{o}} P_{d}^{\beta}\left(r_{x}\right)\left|h_{x, d(\hat{o})}\right|^{2} r_{x, d(\hat{o})}^{-\alpha}$ and $I_{O U}(\epsilon)=\sum_{x \in \Phi_{b} \backslash \hat{o}} P_{u}^{\epsilon}\left(r_{u x}\right)\left|h_{x, d(\hat{o})}\right|^{2} r_{x, d(\hat{o})}^{-\alpha}$ are other BS downlink interference and uplink UE interferences. It should be noted that here, the interference from other base stations is a function of $\beta$.

Theorem 5. The coverage probability of a downlink user in full duplex cellular network with fractional uplink and downlink power control is given by

$$
\begin{aligned}
\mathbb{P}_{c, D L}^{\epsilon, \beta}(T) & \approx \mathbb{E}_{r_{d}, l}\left[F_{\epsilon}^{1}\left(\Lambda_{\lambda, \alpha}^{T}\left(\frac{P_{u}^{\circ}}{P_{d}^{\circ}}\right)^{\frac{2}{\alpha}} R^{2 \epsilon} r_{d}^{2(1-\beta)}\right)\right. \\
& \left.\times F_{\frac{\alpha \epsilon}{2}}^{2}\left(\frac{T P_{u}^{\circ} R^{\alpha \epsilon} r_{d}^{\alpha(1-\beta)}}{P_{d}^{\circ} l^{\alpha}}\right) F_{\beta}^{1}\left(\Lambda_{\lambda, \alpha}^{T} R^{2 \beta} r_{d}^{2(1-\beta)}\right) e^{-\frac{T r_{d}^{\alpha(1-\beta)} \sigma^{2}}{P_{d}^{\circ}}}\right],
\end{aligned}
$$

where the expectation is over $r_{d}$ and $l$, and their pdf is given in (5), and (6). 
Proof.

$$
\begin{aligned}
& \left.P_{c}^{\epsilon, \beta}(T)\right|_{r_{d}, r_{u}, l} \\
& \stackrel{(a)}{=} \mathbb{P}\left[e^{\left.-\frac{T r_{d}^{\alpha}}{P_{d}^{\beta}\left(r_{d}\right)}\left(\sigma^{2}+P_{u}^{\epsilon}\left(r_{u}\right)\left|h_{u}\right|^{2} l^{-\alpha}+I_{O B}(\beta)+I_{O U}(\epsilon)\right)\right]},\right. \\
& \stackrel{(b)}{=}\left(\frac{e^{-\frac{T_{d}^{\alpha} \sigma_{d}^{2}}{P_{d}^{\circ} r_{d}^{\alpha \beta}}}}{1+\frac{T P_{u}^{\circ} r_{d}^{\alpha(1-\beta)} r_{u}^{\alpha \epsilon}}{P_{d}^{\circ} l^{\alpha}}}\right) \mathcal{L}_{O B}^{(\beta)}\left(\frac{T r_{d}^{\alpha}}{P_{d}^{\circ} r_{d}^{\alpha \beta}}\right) \mathcal{L}_{O U}^{(\epsilon)}\left(\frac{T r_{d}^{\alpha}}{P_{d}^{\circ} r_{d}^{\alpha \beta}}\right), \\
& \stackrel{(c)}{=} \exp \left(-\frac{T r_{d}^{\alpha(1-\beta)} \sigma^{2}}{P_{d}^{\circ}}\right){ }_{2} F_{1}\left(1, \frac{2}{\alpha \epsilon} ; \frac{\alpha \epsilon+2}{\alpha \epsilon} ;-\frac{T P_{u}^{\circ} R^{\alpha \epsilon} r_{d}^{\alpha(1-\beta)}}{P_{d}^{\circ} l^{\alpha}}\right) \times \\
& { }_{1} F_{1}\left(\frac{1}{\beta} ; \frac{\beta+1}{\beta} ;-\Lambda_{\lambda, \alpha}^{T} R^{2 \beta} r_{d}^{2(1-\beta)}\right){ }_{1} F_{1}\left(\frac{1}{\epsilon} ; \frac{\epsilon+1}{\epsilon} ;-\Lambda_{\lambda, \alpha}^{T}\left(\frac{P_{u}^{\circ}}{P_{d}^{\circ}}\right)^{\frac{2}{\alpha}} R^{2 \epsilon} r_{d}^{2(1-\beta)}\right) .
\end{aligned}
$$

where $(a)$ follows as $\left|h_{\hat{o}}\right|^{2}$ is exponential random variable and using its tail probability, $(b)$ is by using the the definition of Laplace transform and $(c)$ is by taking the expecation over $r_{u}$ with pdf given in (5). The derivation of Laplace transforms of interference is similar to those in (23) and (24). The proof is complete by taking the expectation of (32) over $r_{d}$ and $l$.

2) Uplink Coverage: Conditioned that, the user is at a distance $r_{u}$ from the parent $\mathrm{BS}$, the SINR is given by

$$
\left.\operatorname{SINR}\right|_{r_{u}}=\frac{P_{u}^{\epsilon}\left(r_{u}\right)\left|h_{u(\hat{o}), \hat{o}}\right|^{2} r_{u}^{-\alpha}}{\sigma^{2}+I_{O B}(\beta)+I_{O U}(\epsilon)}
$$

where $I_{O B}(\beta)$ and $I_{O U}(\epsilon)$ are respectively the downlink and uplink interference from other cells.

Theorem 6. Coverage probability of an uplink user in full duplex network with fractional uplink power control and downlink power control is given by

$$
\mathbb{P}_{c, U L}^{\epsilon, \beta}(T) \approx \mathbb{E}_{r_{u}}\left[F_{\beta}^{1}\left(\Lambda_{\lambda, \alpha}^{T}\left(\frac{P_{d}^{\circ}}{P_{u}^{\circ}}\right)^{\frac{2}{\alpha}} R^{2 \beta} r_{u}^{2(1-\epsilon)}\right) F_{\epsilon}^{1}\left(\Lambda_{\lambda, \alpha}^{T} r_{u}^{2(1-\epsilon)} R^{2 \epsilon}\right) e^{-\frac{\sigma^{2} T r_{u}^{\alpha(1-\epsilon)}}{P_{u}^{\circ}}}\right],
$$

where the expectation is over the random variable $r_{u}$ and it's pdf is given in (5).

Proof. Follows the similar procedure of theorem (5)

\section{A. Results and Discussions}

The coverage probabilities with power controls in both uplink and downlink are plotted in Fig. 6. The simulations are in close agreement with the analytical results. As a direct result of 
downlink power control, uplink coverage is enhanced by reducing $\beta$ but with slight degradation of downlink coverage. We can also see that for a given $\epsilon$, increasing $\beta$ enhances downlink coverage, but decreases uplink coverage, and vice versa. In Fig. 7, the dependency of density of BSs and coverage are plotted for various combinations of $(\epsilon, \beta)$. It can be seen that the uplink coverage probability can be improved, even in high dense networks by adjusting the parameters $\beta$ and $\epsilon$, whereas this was not possible in FD networks with constant downlink power.

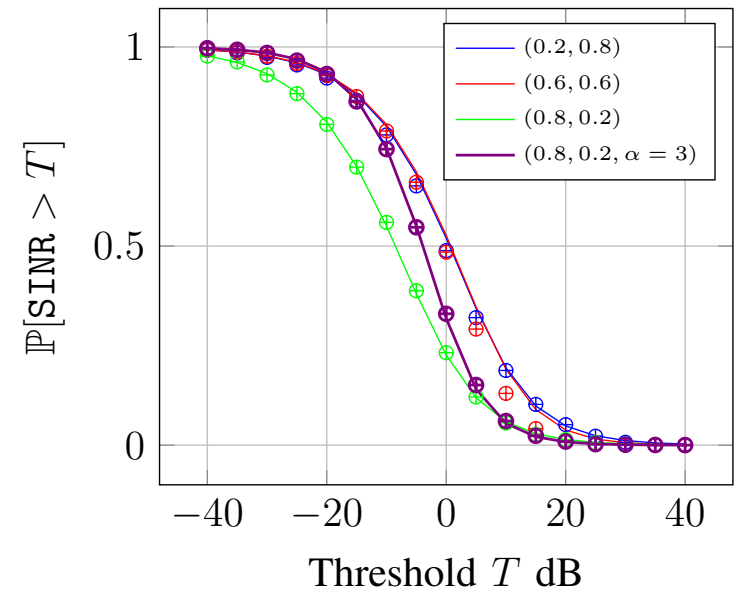

(a) Downlink Coverage

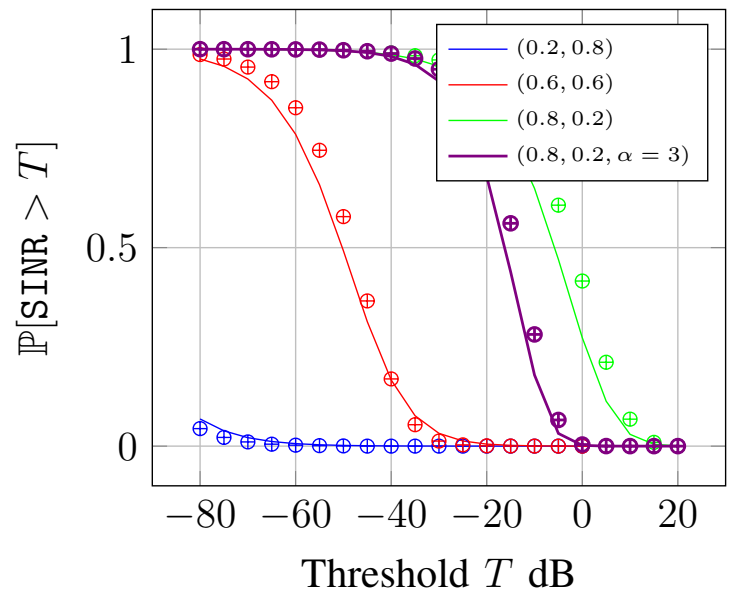

(b) Uplink Coverage

Fig. 6: Coverage probability of a typical user in FD for various configuration of $(\epsilon, \beta)$ with $P_{d}^{\circ}=-13 \mathrm{dBm}, P_{u}^{\circ}=-64 \mathrm{dBm}$ and cell radius, $R=200 \mathrm{~m}$. Simulations are marked by respective coloured $\oplus$.

The rate CDFs for various configuration of $(\epsilon, \beta)$ are plotted in Fig. 8. We can see that the uplink rate CDFs are aligned more towards the right side of the graph (towards the higher rate regions) for small values of $\beta$ and higher values of $\epsilon$, compared with constant power downlink transmissions. But this degrades the downlink rates. The extend of degradation or improvements in downlink performance depends also on the other parameters like density, $P_{d}^{\circ}$, $P_{0}^{\circ}$ and environment $(\alpha)$. Thus, there is a trade-off between downlink and uplink rates when using both uplink and downlink power control. Taking into account the dependence of rates with other system parameters as well, network design becomes a multivariate optimization problem. In the following section, we will discuss the optimization of average rates in FD networks. 


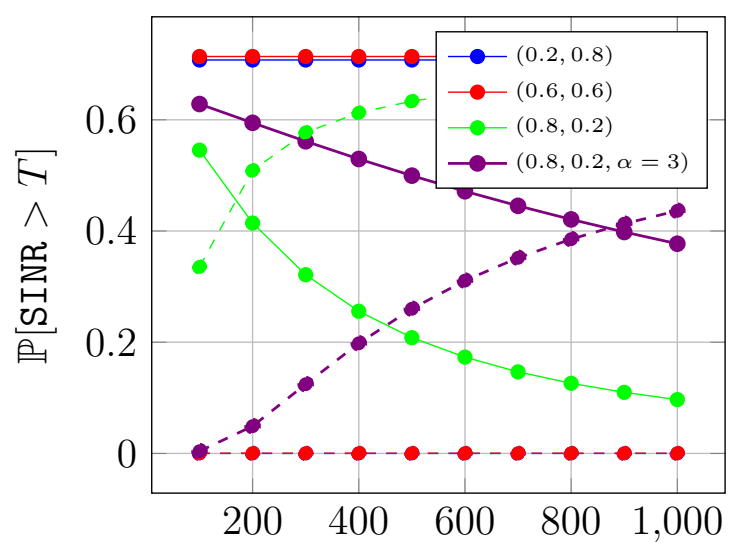

Cell radius $R$ in $\mathrm{m}$

Fig. 7: Coverage probability Vs cell radius for various $(\epsilon, \beta)$ configurations, $P_{u}^{\circ}=-64 \mathrm{dBm}$, $P_{d}^{\circ}=-13 \mathrm{dBm}$ at a threshold $-6 \mathrm{~dB}$. Solid curves for the downlink and dashed for the uplink.

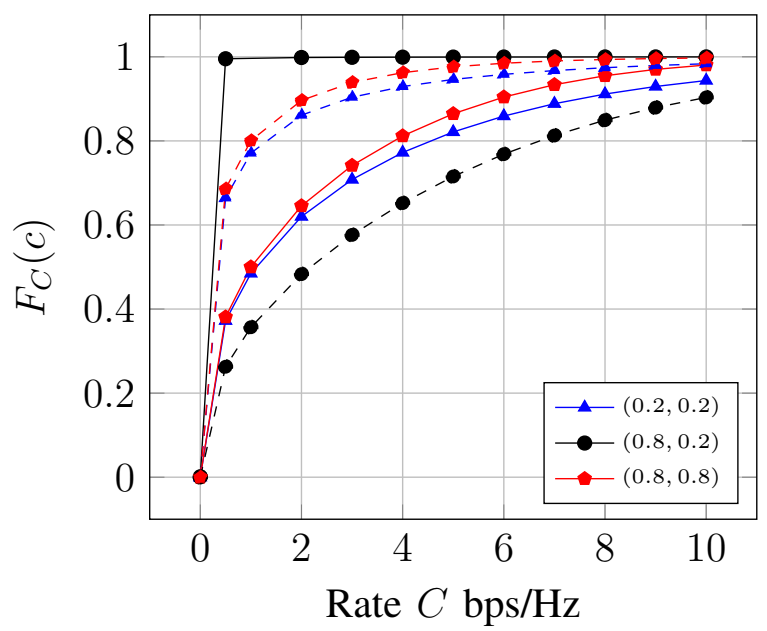

Fig. 8: Rate CDF of a typical uplink/downlink user in FD with uplink and downlink power control for various configuration $\epsilon$ and $\beta,(\epsilon, \beta)$ with $P_{u}^{\circ}=-64 \mathrm{dBm}, P_{d}^{\circ}=-54 \mathrm{dBm}$ and cell radius, $R=200 \mathrm{~m}$. Dashed line represents uplink and solid lines for downlink.

\section{Optimal $(\epsilon, \beta)$ And a Distance Based Scheduling}

The mean rate and cell edge rates are plotted for various values of $(\epsilon, \beta)$ as a surface plot in Fig. 9. We can see that the average uplink or downlink rates can be as high as about 4.5 $\mathrm{bps} / \mathrm{Hz}$, whereas the conventional HD rates are about $2.02 \mathrm{bps} / \mathrm{Hz}$. Likewise, cellular edge rates can reach 0.03 in uplink or downlink. From the surface plots, we can see that maximizing both downlink and uplink cannot be done together, we are therefore trying to obtain combinations of 


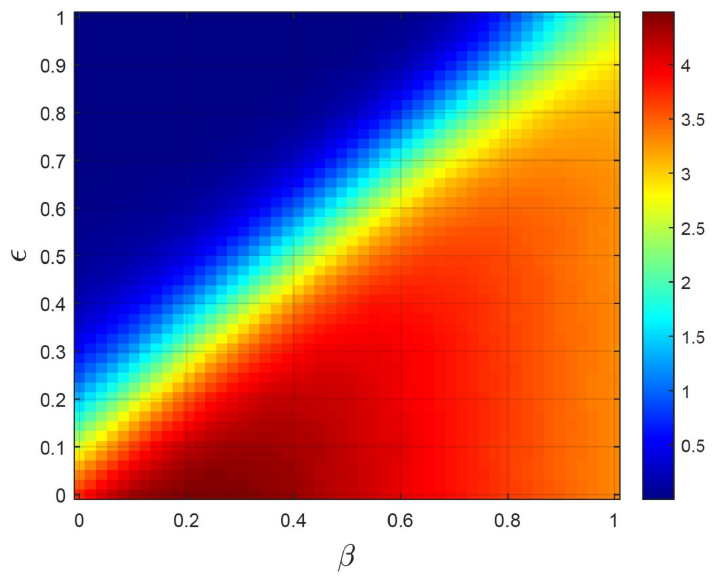

(a) Downlink mean rate

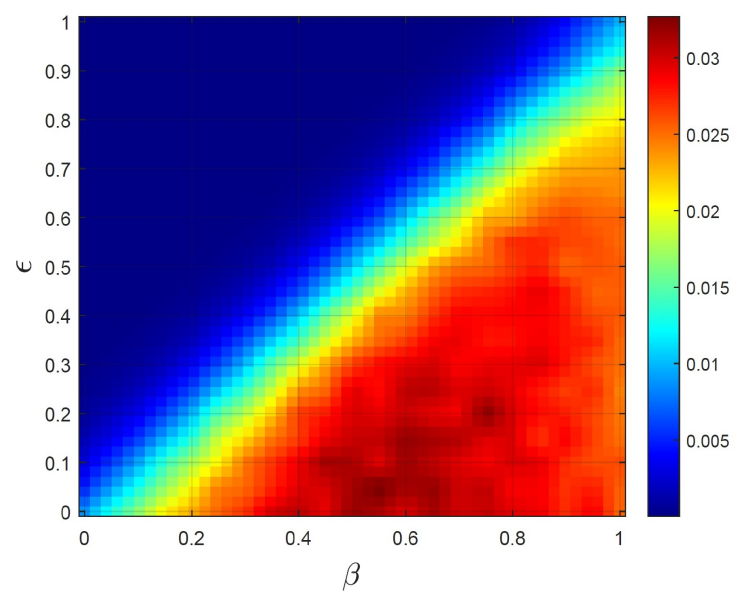

(c) Downlink cell edge rate

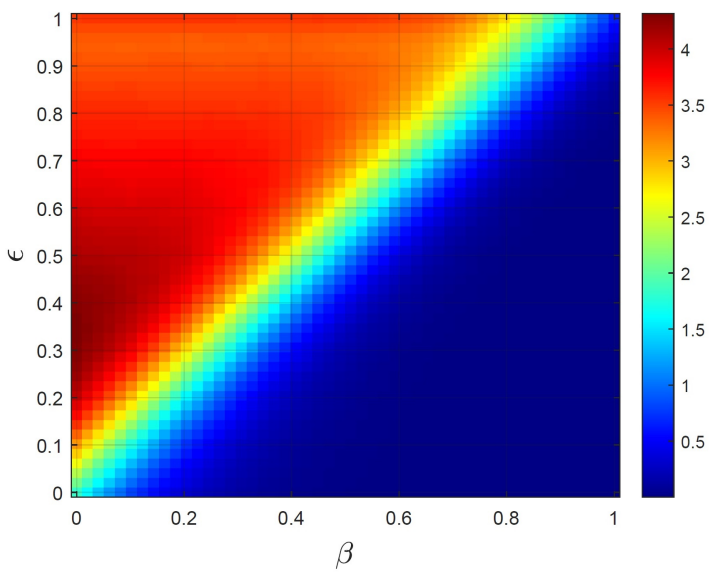

(b) Uplink mean rate

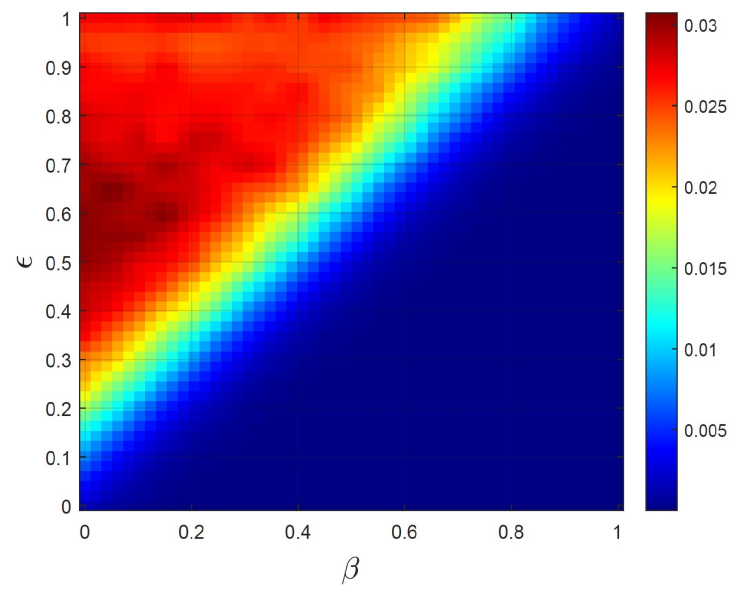

(d) Uplink cell edge rate

Fig. 9: Surface plot of mean and cell edge rate against diffrent power control vaues $\epsilon$ and $\beta$ with $P_{0}^{\circ}=-64 \mathrm{dBm}, P_{d}^{\circ}=-54 \mathrm{dBm}$, and cell radius $200 \mathrm{~m}$.

$(\epsilon, \beta)$ such that the uplink and downlink rates present improvements compared to HD systems.

We can see from Fig. 9, the mean rate regions of the range $(0.2 \leq \beta, \epsilon \leq 0.3)$ privide a mean rate of $2.7 \mathrm{bps} / \mathrm{Hz}$ in downlink and $2.3 \mathrm{bps} / \mathrm{Hz}$ in uplink (yellow-colored regions). The cell edge rate for this region is in the range of 0.01 for both downlink and uplink. To mutually benefit from downlink power control, downlink and uplink users have sacrificed part of their achievable rates. Therefore, to further enhance performance, a distance-based user scheduling is considered. In this scheduling, two users are permitted to use the same frequency if they are separated by a minimum distance $D$. Fig. 10 depicts a comparison study for a system with and without this scheduling. It can be observed that when the minimum distance between users sharing the same 
resource elements increases, the average rate and the cell edge rate increase. With this scheme in uplink, cell edge rate improved to $0.03 \mathrm{bps} / \mathrm{Hz}$ from $10^{-4}$ and mean rate improved to $3 \mathrm{bps} / \mathrm{Hz}$ from $0.15 \mathrm{bps} / \mathrm{Hz}$. In the downlink, the mean rate increased from $2.02 \mathrm{bps} / \mathrm{Hz}$ to $3 \mathrm{bps} / \mathrm{Hz}$ and the cell edge rate increased to 0.03 from $0.005 \mathrm{bps} / \mathrm{Hz}$ over $\mathrm{HD}$ networks. These results suggest that downlink power control can provide better FD performance in downlink and uplink of cellular networks.

We limited the optimization variables only to $\epsilon$ and $\beta$ while maintaining the other parameters fixed, the rate optimization for all design-parameters will be an interesting extension of this work. Moreover, we believe that there may be better power control policies other than the fractional power control contemplated in this paper.

\section{CONCLUSION}

In this paper, we characterized the performance of FD cellular networks. We modeled BS-UE interactions using Matèrn clustur process. Expressions of the coverage probability of a typical user in downlink and uplink are derived. We have considered three different transmit power scenarios. We have shown that FD with constant downlink transmit power will hurt the uplink users and cell edge users. We proposed a fractional power control in downlink transmission and showed that the performance of uplink users can be improved, but with reduced downlink rates. This reduction in downlink rate can be compensated by a distance based user scheduling. We also showed that performance can be optimized for better downlink and uplink performance when compared to HD and FD without downlink power control. In this paper, the optimization and scheduling are carried out for mean and cell edge rate performances with only $(\epsilon, \beta)$ as design parameters, the optimization with other parameters will be an interesting extension of this work.

\section{REFERENCES}

[1] J. G. Andrews, S. Buzzi, W. Choi, S. V. Hanly, A. Lozano, A. C. K. Soong, and J. C. Zhang, "What will 5G be?” IEEE Journal on Selected Areas in Communications, vol. 32, no. 6, pp. 1065-1082, 2014.

[2] R. ITU-R, "IMT vision-framework and overall objectives of the future development of IMT for 2020 and beyond," Sep. 2015.

[3] Z. Zhang, Y. Xiao, Z. Ma, M. Xiao, Z. Ding, X. Lei, G. K. Karagiannidis, and P. Fan, "6G wireless networks: Vision, requirements, architecture, and key technologies,” IEEE Vehicular Technology Magazine, vol. 14, no. 3, pp. 28-41, 2019.

[4] ITU-R, "IMT traffic estimates for the years 2020 to 2030," in Report ITU-R M. 2370-0, ITU-R Radiocommunication Sector of ITU, 2015. 


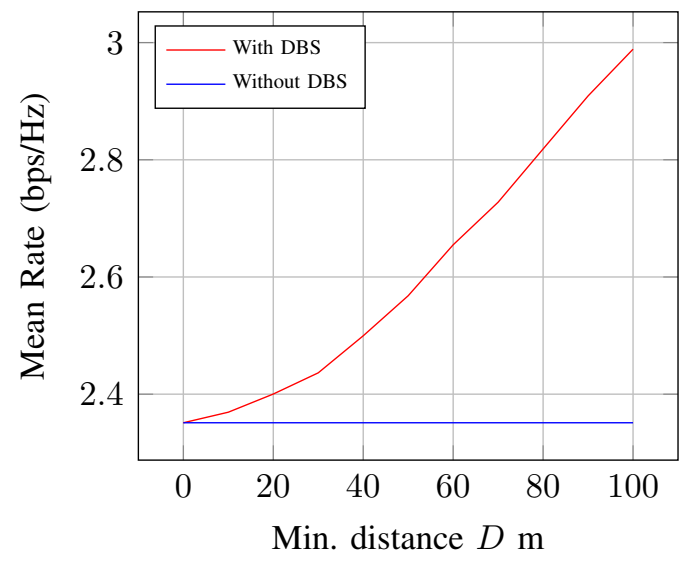

(a) Downlink mean rate

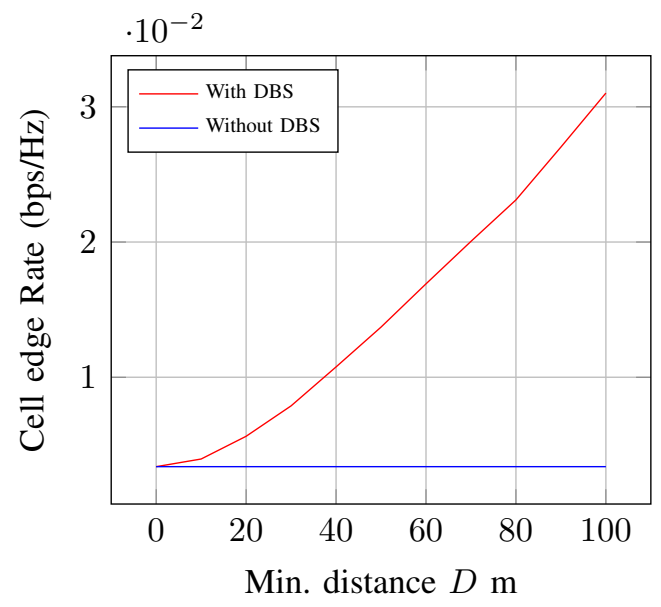

(c) Downlink cell edge rate

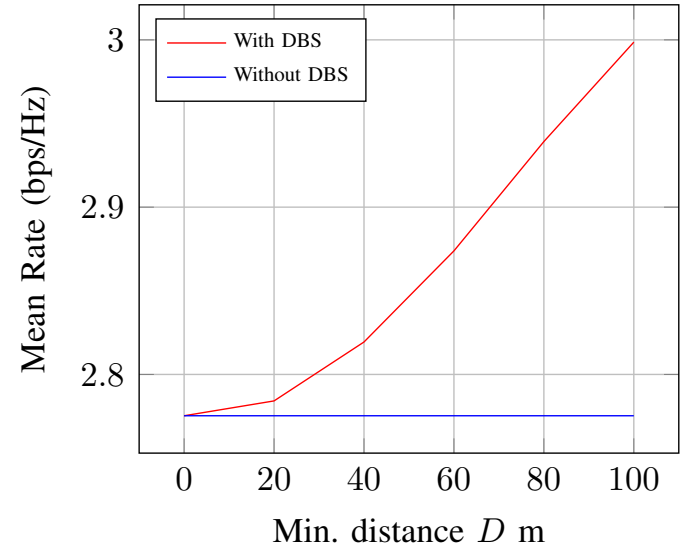

(b) Uplink mean rate

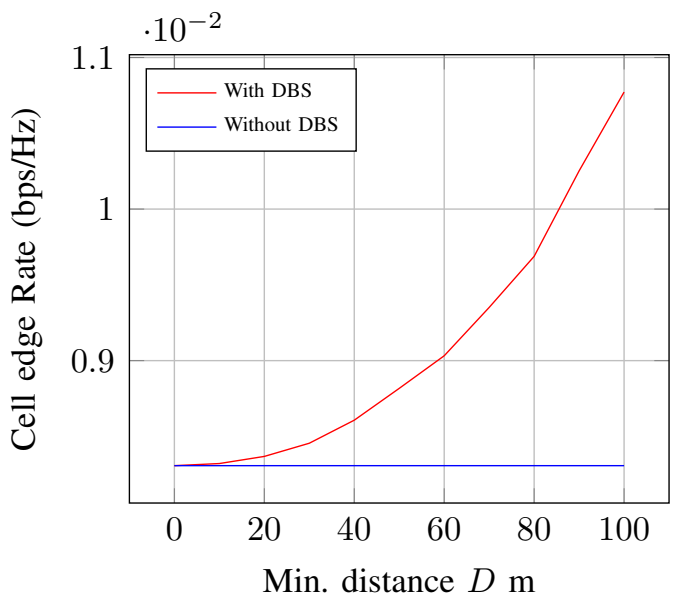

(d) Uplink cell edge rate

Fig. 10: Mean and cell edge rate against minimum distance $D$ for DBS with $\epsilon=0.3, \beta=0.2$, $P_{0}^{\circ}=-64 \mathrm{dBm}, P_{d}^{\circ}=-54 \mathrm{dBm}$ and cell radius $200 \mathrm{~m}$.

[5] H. ElSawy, A. AlAmmouri, O. Amin, and M. Alouini, "Can uplink transmissions survive in full-duplex cellular environments?" in European Wireless 2016; 22th European Wireless Conference, May 2016, pp. 1-6.

[6] Z. Tong and M. Haenggi, "Throughput analysis for full-duplex wireless networks with imperfect self-interference cancellation,” IEEE Transactions on Communications, vol. 63, no. 11, pp. 4490-4500, Nov 2015.

[7] C. D. Nwankwo, L. Zhang, A. Quddus, M. A. Imran, and R. Tafazolli, “A survey of self-interference management techniques for single frequency full duplex systems," IEEE Access, vol. 6, pp. 30242-30268, 2018.

[8] Z. Zhang, X. Chai, K. Long, A. V. Vasilakos, and L. Hanzo, "Full duplex techniques for 5G networks: self-interference cancellation, protocol design, and relay selection," IEEE Communications Magazine, vol. 53, no. 5, pp. 128-137, 2015.

[9] A. Sabharwal, P. Schniter, D. Guo, D. W. Bliss, S. Rangarajan, and R. Wichman, "In-band full-duplex wireless: Challenges and opportunities," IEEE Journal on Selected Areas in Communications, vol. 32, no. 9, pp. 1637-1652, 2014. 
[10] S. Goyal, C. Galiotto, N. Marchetti, and S. Panwar, "Throughput and coverage for a mixed full and half duplex small cell network," in IEEE ICC, May 2016, pp. 1-7.

[11] T. Chen, J. Diakonikolas, J. Ghaderi, and G. Zussman, "Hybrid scheduling in heterogeneous half-and full-duplex wireless networks," in IEEE INFOCOM 2018 - IEEE Conference on Computer Communications, April 2018, pp. 576-584.

[12] J. Lee and T. Q. S. Quek, "Hybrid full-/half-duplex system analysis in heterogeneous wireless networks," IEEE Transactions on Wireless Communications, vol. 14, no. 5, pp. 2883-2895, May 2015.

[13] M. O. Al-Kadri, Y. Deng, A. Aijaz, and A. Nallanathan, "Full-duplex small cells for next generation heterogeneous cellular networks: A case study of outage and rate coverage analysis," IEEE Access, vol. 5, pp. 8025-8038, 2017.

[14] L.-E. U. terrestrial Radio Access(E-UTRA), "Physical layer procedures," 3GPP TS 36.213 version 8.8.0 Release 8, 2010.

[15] C. Saha, M. Afshang, and H. S. Dhillon, "3GPP-inspired HetNet model using poisson cluster process: Sum-product functionals and downlink coverage," IEEE Transactions on Communications, vol. 66, no. 5, pp. 2219-2234, May 2018.

[16] — - "Poisson cluster process: Bridging the gap between PPP and 3GPP HetNet models," in 2017 Information Theory and Applications Workshop (ITA), Feb 2017, pp. 1-9.

[17] M. Afshang, C. Saha, and H. S. Dhillon, "Nearest-neighbor and contact distance distributions for Matèrn Cluster Process," IEEE Communications Letters, vol. 21, no. 12, pp. 2686-2689, Dec 2017.

[18] Y. Yao, B. Li, C. Li, C. Yang, and B. Xia, "Downlink performance analysis of the full-duplex networks with interference cancellation," IEEE Transactions on Communications, vol. 68, no. 4, pp. 2324-2338, 2020.

[19] A. M. Kundu, R. Pal, M. Kumar, and T. V. Sreejith, "Uplink and downlink performance bounds for full duplex cellular networks," in 2020 IEEE International Black Sea Conference on Communications and Networking (BlackSeaCom), 2020, pp. 1-6.

[20] I. Randrianantenaina, H. ElSawy, H. Dahrouj, M. Kaneko, and M. Alouini, "Uplink power control and ergodic rate characterization in FD cellular networks: A stochastic geometry approach,” IEEE Transactions on Wireless Communications, vol. 18, no. 4, pp. 2093-2110, April 2019.

[21] B. Li, C. Li, C. Yang, and B. Xia, "User scheduling for full-duplex cellular networks," in 2019 IEEE International Conference on Communications Workshops (ICC Workshops), 2019, pp. 1-6.

[22] R. Kiran and N. B. Mehta, "Reduced feedback, user scheduling, and mode selection in asymmetric full-duplex systems," in GLOBECOM 2020 - 2020 IEEE Global Communications Conference, 2020, pp. 1-6.

[23] _ _ "User-pair scheduling and mode selection in asymmetric full-duplex systems with limited feedback: Algorithm and scaling laws," IEEE Transactions on Wireless Communications, vol. 20, no. 5, pp. 2863-2875, 2021.

[24] J. Tang, G. Chen, J. P. Coon, and D. E. Simmons, "Distance distributions for Matèrn cluster processes with application to network performance analysis," in 2017 IEEE International Conference on Communications (ICC), 2017, pp. 1-6.

[25] J. G. Andrews, F. Baccelli, and R. K. Ganti, "A tractable approach to coverage and rate in cellular networks," IEEE Transactions on Communications, vol. 59, no. 11, pp. 3122-3134, November 2011.

[26] A. Shojaeifard, K. Wong, W. Yu, G. Zheng, and J. Tang, "Full-duplex cloud radio access network: Stochastic design and analysis," IEEE Transactions on Wireless Communications, vol. 17, no. 11, pp. 7190-7207, 2018.

[27] Ericsson, "R1-074850: uplink power control for E-UTRA-range and representation of P0," 3GPP TSG RAN WG1 Meeting \#51, Nov 2007.

[28] A. Simonsson and A. Furuskar, "Uplink power control in LTE - overview and performance, subtitle: Principles and benefits of utilizing rather than compensating for SINR variations," 2008 IEEE 68th Vehicular Technology Conference, pp. 1-5, September 2008 . 